\title{
Video Article \\ Quantitative Assessment of Immune Cells in the Injured Spinal Cord Tissue by Flow Cytometry: a Novel Use for a Cell Purification Method
}

\author{
Hal X. Nguyen ${ }^{1,2,3,4}$, Kevin D. Beck ${ }^{5}$, Aileen J. Anderson ${ }^{1,2,3,4,6}$ \\ ${ }^{1}$ Institute for Memory Impairments and Neurological Disorders, University of California \\ ${ }^{2}$ Physical Medicine \& Rehabilitation, University of California \\ ${ }^{3}$ Anatomy \& Neurobiology, University of California \\ ${ }^{4}$ Sue and Bill Gross Stem Cell Research Center, University of California \\ ${ }^{5}$ Section of Molecular Biology, University of California \\ ${ }^{6}$ Reeve-Irvine Research Center, University of California
}

Correspondence to: Hal X. Nguyen at hnguyen@uci.edu, Aileen J. Anderson at aja@uci.edu

URL: https://www.jove.com/video/2698

DOI: doi:10.3791/2698

Keywords: Immunology, Issue 50, spinal cord injury, cellular inflammation, neuroinflammation, OptiPrep, central nervous system, neutrophils, macrophages, microglia, T-cells, flow cytometry

Date Published: 4/9/2011

Citation: Nguyen, H.X., Beck, K.D., Anderson, A.J. Quantitative Assessment of Immune Cells in the Injured Spinal Cord Tissue by Flow Cytometry: a Novel Use for a Cell Purification Method. J. Vis. Exp. (50), e2698, doi:10.3791/2698 (2011).

\section{Abstract}

Detection of immune cells in the injured central nervous system (CNS) using morphological or histological techniques has not always provided true quantitative analysis of cellular inflammation. Flow cytometry is a quick alternative method to quantify immune cells in the injured brain or spinal cord tissue. Historically, flow cytometry has been used to quantify immune cells collected from blood or dissociated spleen or thymus, and only a few studies have attempted to quantify immune cells in the injured spinal cord by flow cytometry using fresh dissociated cord tissue. However, the dissociated spinal cord tissue is concentrated with myelin debris that can be mistaken for cells and reduce cell count reliability obtained by the flow cytometer. We have advanced a cell preparation method using the OptiPrep gradient system to effectively separate lipid/ myelin debris from cells, providing sensitive and reliable quantifications of cellular inflammation in the injured spinal cord by flow cytometry. As described in our recent study (Beck \& Nguyen et al., Brain. 2010 Feb; 133 (Pt 2): 433-47), the OptiPrep cell preparation had increased sensitivity to detect cellular inflammation in the injured spinal cord, with counts of specific cell types correlating with injury severity. Critically, novel usage of this method provided the first characterization of acute and chronic cellular inflammation after SCl to include a complete time course for polymorphonuclear leukocytes (PMNs, neutrophils), macrophages/microglia, and T-cells over a period ranging from 2 hours to 180 days post-injury (dpi), identifying a surprising novel second phase of cellular inflammation. Thorough characterization of cellular inflammation using this method may provide a better understanding of neuroinflammation in the injured CNS, and reveal an important multiphasic component of neuroinflammation that may be critical for the design and implementation of rational therapeutic treatment strategies, including both cell-based and pharmacological interventions for SCl.

\section{Video Link}

The video component of this article can be found at https://www.jove.com/video/2698/

\section{Dissociation of spinal cord tissue}

1. Spinal cord segments T8-T10 of non-injured or contusion spinal cord injured (injury at T9) Sprague Dawley rats were dissected and mechanically dissociated with fine scissors in HBSS at room temperature, as previously described ${ }^{1}$. Prior to tissue dissociation, whole spinal cord columns were kept on dry ice for 5 minutes before the extraction of cord segments T8-T10.

2. Tissue bits were retrieved by centrifugation ( 1 minute, $1000 \mathrm{rpm}$, room temperature) and enzymatically dissociated with $2.5 \mathrm{mg}$ trypsin and 5 $\mathrm{mg}$ collagenase in $5 \mathrm{ml}$ DME (Dulbecco's Modified Eagle's Media) for 20 minutes at $37^{\circ} \mathrm{C}$ before trituration ( 10 times at room temperature) with a glass Pasteur pipette (9-inches).

3. $10 \mathrm{ml}$ of DME $+10 \%$ fetal bovine serum was added to cells to inhibit enzymatic activities and then was filtered through a $40 \mu \mathrm{m}$ cell strainer. After a quick spin, the cell pellet was resuspended in $6 \mathrm{ml}$ of HBSS and overlayed on OptiPrep gradient solutions described below.

\section{Creating OptiPrep gradient solutions}

1. Diluted OptiPrep was constructed by diluting OptiPrep 1:1 with MOPS Buffer (0.15 M NaCl, $10 \mathrm{mM} \mathrm{MOPS}, \mathrm{pH} 7.4)$. 
2. Four OptiPrep gradient solutions were made by mixing 350, 250, 200, or $150 \mu$ l diluted OptiPrep with HBSS to a final volume of $1 \mathrm{ml}$ (Table 1).

3. The four solutions were slowly and carefully placed in layers in a $15 \mathrm{ml}$ conical tube with the least dilute at the bottom and the most dilute on the top (Figure 1A).

\section{Separating lipid/myelin debris from cells}

1. $6 \mathrm{ml}$ of dissociated spinal cells in HBSS was carefully layered on top of the OptiPrep gradient solutions.

2. Tube containing cells and gradient solutions was centrifuged ( 15 minutes, $1900 \mathrm{rpm}$ or $726 \mathrm{RCF}, 20^{\circ} \mathrm{C}$ ) using an Eppendorf Centrifuge $5810 \mathrm{R}$ with a swing-bucket rotor (A-4-62), separating the cell solution into distinct layers with lipid/myelin debris (top $7 \mathrm{ml}$ of tube), followed by 3 layers of neurons, with inflammatory cells, glia, and red blood cells in the pellet. Although most inflammatory cells, including monocytes, macrophages, PMN granulocytes, and lymphocytes were found in the pellet, some large sized-activated macrophages could be found in layers above the pellet.

3. The lipid/myelin debris layer (top $7 \mathrm{ml}$ ) was carefully aspirated and removed. Cells were then washed and resuspended in $2.5 \mathrm{ml}$ HBSS and used for immunolabeling below.

\section{Immunolabeling of specific immune cells for flow cytometry}

1. Cells $(500 \mu \mathrm{l})$ collected from spinal cord preparations were pelleted and resuspended in $0.85 \%$ ammonium chloride (diluted in distilled water) for 5 minutes to lyse red blood cells.

2. Cells were washed with $500 \mu \mathrm{HBSS}$ and then blocked for 30 minutes in normal rabbit or mouse serum at room temperature.

3. Cells were washed and incubated at room temperature for 1 hour with antibody (Rabbit anti-PMN FITC, Accurate Chemical and Scientific; Mouse anti-rat ED1 Alexa 488, Serotec; or Mouse anti-rat CD3 Alexa 488) or isotype IgG solution diluted in HBSS (all at 1:100 dilution), as described previously ${ }^{1}$.

4. Cells were washed twice after each step above and then resuspended in $300 \mu \mathrm{l} \mathrm{HBSS}$ after the final step.

\section{Cell quantitative assessment by flow cytometry}

1. Samples were analyzed on a FACS Calibur (Becton-Dickinson) flow cytometer using Cell Quest software. 5,000 events per sample were read for all samples, and data analysis was completed with Summit (DakoCytomation).

2. Flow cytometric gates were set using control lgG isotype labelled cells or spinal cord cells from uninjured control animals to set baseline values for normalization across time points as described previously ${ }^{1}$. The mean values of positively labelled cells were expressed as percent $( \pm$ S.E.M.) of the entire sample.

\section{Representative Results:}

The ability of an OptiPrep gradient to remove myelin debris and improve immune cell detection by flow cytometry was assessed by quantifying spinal PMNs at $1 \mathrm{dpi}$ (Figure 1B), the previously reported peak of PMN infiltration after $\mathrm{SCl}^{1-3}$. Alternatively, identically dissected spinal cords were enzymatically dissociated without OptiPrep-removal of myelin debris and were similarly assessed in parallel for PMN infiltration by flow cytometry. As we have reported previously ${ }^{1}$, there was a shift in the position of events in samples isolated with OptiPrep gradient purification method compared to enzymatic dissociation alone, demonstrating the percentage of PMNs detected in samples with intact myelin debris was only a fraction $(0.5 \%)$ of the percentage of PMNs detected $(5.1 \%)$ in OptiPrep purified samples (Figure 1B). These data suggest that myelin debris in tissue preparations can obscure flow cytometric readings, and demonstrate that removal of myelin debris enhances the sensitivity of immune cell detection in the injured spinal cord tissue.

Having established the sensitivity of the OptiPrep gradient system for cell detection in the injured spinal cord, we characterized changes in cellular infiltration over a period ranging from $2 \mathrm{hrs}$ to $180 \mathrm{dpi}$ and established a novel multiphasic response of cellular inflammation after SCl that included PMNs, macrophages/microglia and T-cells. As confirmed by previous studies ${ }^{1-3}$, PMNs first entered the cord at 2 hrs post-injury and peaked at $1 \mathrm{dpi}$ (Figure $2 \& 4$ ). Macrophages/microglia on the other hand ${ }^{1,4,5}$, were not detected in the injured spinal cord until $3 \mathrm{dpi}$, and peaked initially at $7 \mathrm{dpi}$ (Figure $3 \& 4$ ). Surprisingly, we show for the first time that macrophages/microglia peaked for the second time $60 \mathrm{dpi}$ and remained elevated through $180 \mathrm{dpi}$ (Figure $3 \& 4$ ). Similar to macrophages/microglia, T-cell infiltration was predicted to peak $7 \mathrm{dpi}{ }^{5-7}$; however, flow cytometry did not detect any changes in T-cell number in the first $7 \mathrm{dpi}$, though an elevated number of T-cells was detected at $9 \mathrm{dpi}$ (1.6\%) (Figure 4). While T-cell number was decreasing by $10 \mathrm{dpi}$, a persistent T-cell response was observed through $180 \mathrm{dpi}$, at which time $4.4 \%$ of cells were labelled for CD3 (Figure 4).

Together, these data demonstrate a time-dependent multiphasic response of cellular inflammation after SCI (Figure 4); the initial phases of cellular inflammation were comprised of the early peak of PMNs $1 \mathrm{dpi}$ followed by a peak of ED $1+$ macrophages/microglia $7 \mathrm{dpi}$ and T-cells $9 \mathrm{dpi}$, while the later phases were composed of all three cellular populations rising after $14 \mathrm{dpi}$ and persisting through $180 \mathrm{dpi}$, with a notable second peak of macrophages/microglia at $60 \mathrm{dpi}$. This timecourse study and the quantitative data generated by flow cytometry have been validated previously, showing comparable results to data collected from quantitative stereology of immunolabeled spinal cord sections 1 . 


\begin{tabular}{|c|c|c|}
\hline Tube & Diluted OptiPrep $(u \mathrm{l})$ & HBSS $(\mu \mathrm{l})$ \\
\hline 1 & 350 & 650 \\
\hline 2 & 250 & 750 \\
\hline 3 & 200 & 800 \\
\hline 4 & 150 & 850 \\
\hline
\end{tabular}

Table 1. Preparation of OptiPrep gradient for the removal of myelin debris. Four OptiPrep gradient solutions are made using HBSS and diluted OptiPrep (1:1 dilution of OptiPrep and MOPS).

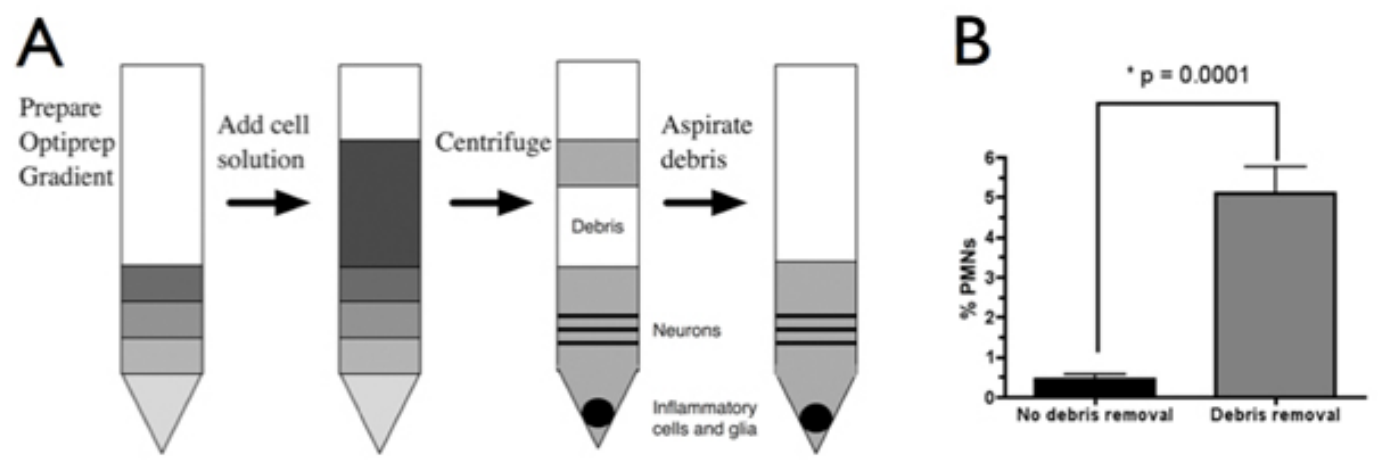

Figure 1. OptiPrep gradient densities separate most myelin/debris from injured spinal cord tissues/cells and improve immune cell assessment by flow cytometry. (A) Myelin/debris was separated from cells (neurons, glia and immune cells) after centrifugation of dissociated spinal cord tissue through an OptiPrep gradient followed by aspiration of myelin/debris layer. (B) PMN number in the injured spinal cord, showing increased sensitivity in detecting PMNs after debris removal (Student's t-test, $p=0.0001$ ). All flow cytometric gates were set using labeled cells from uninjured animals; $n=5$ per group, mean \pm SEM. 5,000 events per sample were read for all samples and the mean values of positively labeled cells were expressed as percent ( \pm S.E.M.) of the entire sample.
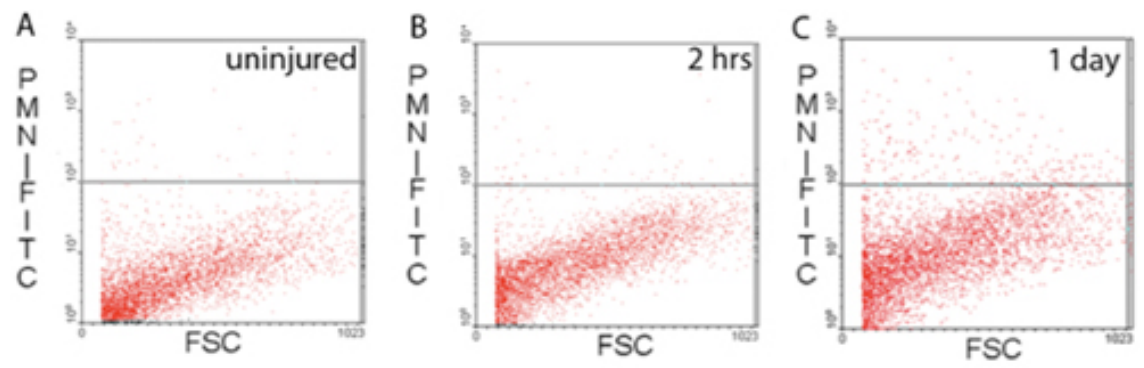

Figure 2. Detection of PMN infiltration in the injured spinal cord by flow cytometry. After a moderate (200 kd) contusion injury at T9, PMNs quickly entered the spinal cord starting $2 \mathrm{hrs}(\mathrm{B})$ post-injury and peaking $1 \mathrm{dpi}(\mathrm{C})$. All flow cytometric gates were set using labeled cells from uninjured animals $(A)$. 

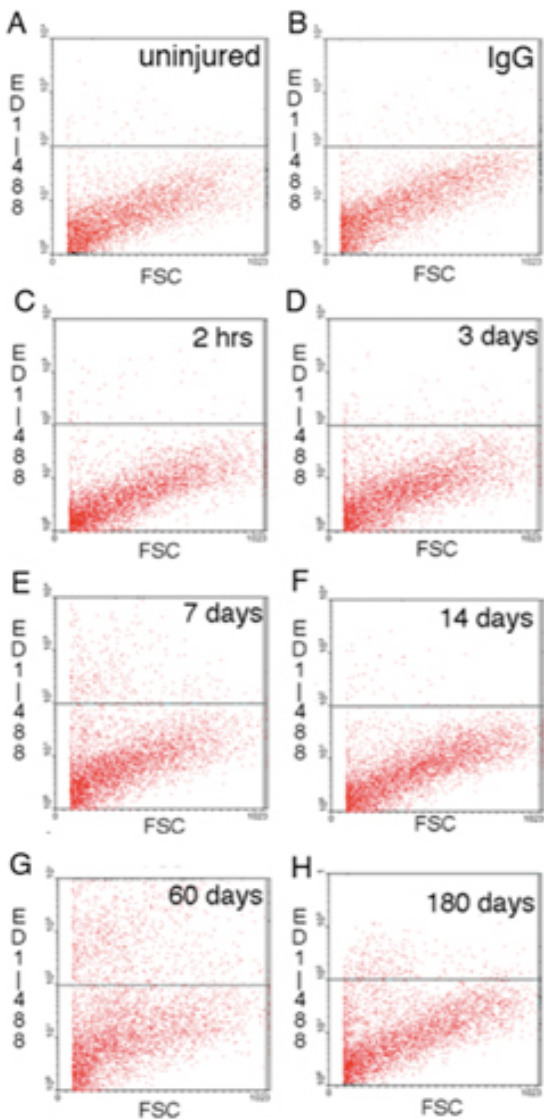

Figure 3. Detection of macrophages/microglia infiltration in the injured spinal cord by flow cytometry. After a moderate (200 kd) contusion injury at T9, ED1+ macrophage/microglial number increased in the injured spinal cord starting at $3 \mathrm{dpi}(\mathrm{D})$, peaked acutely at $7 \mathrm{dpi}(\mathrm{E})$, dropped to low levels at $14 \mathrm{dpi}(\mathrm{F})$, before rising to a second peak at $60 \mathrm{dpi}(\mathrm{G})$, and remained in the injured spinal cord up to $180 \mathrm{dpi}(\mathrm{H})$. IgG isotype control labeling of 7 dpi spinal cells (B) showed minimal antibody-labeling background and no difference to antibody-labeled cells from 2 hrs post-injured $(C)$ or uninjured $(A)$ animals. All flow cytometric gates were set using labeled cells from uninjured animals.

\begin{tabular}{|r|c|c|c|}
\hline $\begin{array}{c}\text { Table 2: Animal samples in } \\
\text { timecourse experiments }\end{array}$ & PMN & ED1 & CD3 \\
\hline $0 \mathrm{hr}$ & 5 & 5 & 5 \\
\hline $2 \mathrm{hr}$ & 5 & 5 & 4 \\
\hline $1 \mathrm{dpi}$ & 4 & 5 & 5 \\
\hline $2 \mathrm{dpi}$ & 5 & 3 & 5 \\
\hline $3 \mathrm{dpi}$ & 5 & 3 & 5 \\
\hline $4 \mathrm{dpi}$ & 4 & 5 & 5 \\
\hline $5 \mathrm{dpi}$ & 5 & 5 & 5 \\
\hline $6 \mathrm{dpi}$ & 5 & 5 & 5 \\
\hline $7 \mathrm{dpi}$ & 4 & 5 & 4 \\
\hline $8 \mathrm{dpi}$ & 5 & 5 & 5 \\
\hline $9 \mathrm{dpi}$ & 5 & 5 & 5 \\
\hline $10 \mathrm{dpi}$ & 5 & 5 & 5 \\
\hline $14 \mathrm{dpi}$ & 5 & 5 & 4 \\
\hline $21 \mathrm{dpi}$ & 5 & 5 & - \\
\hline $28 \mathrm{dpi}$ & 4 & 5 & - \\
\hline $60 \mathrm{dpi}$ & 5 & 5 & - \\
\hline $90 \mathrm{dpi}$ & 4 & 4 & 4 \\
\hline $180 \mathrm{dpi}$ & 5 & 5 & 5 \\
\hline & & & \\
\hline & & & \\
\hline & & \\
\hline
\end{tabular}


Table 2. Animal samples in timecourse experiments. For each time point ( $0 \mathrm{hr}$ to $180 \mathrm{dpi})$, five animals received a moderate (200 kd) contusion injury at T9, and spinal cord tissues were assessed by flow cytometry for the numbers of PMNs, ED $1^{+}$macrophages $/$microglia, and $\mathrm{CD}^{+}$T-cells. However, not all animal samples were recovered successfully for PMN (4-5), ED1 (3-5), and CD3 (4-5) flow cytometric analyses.

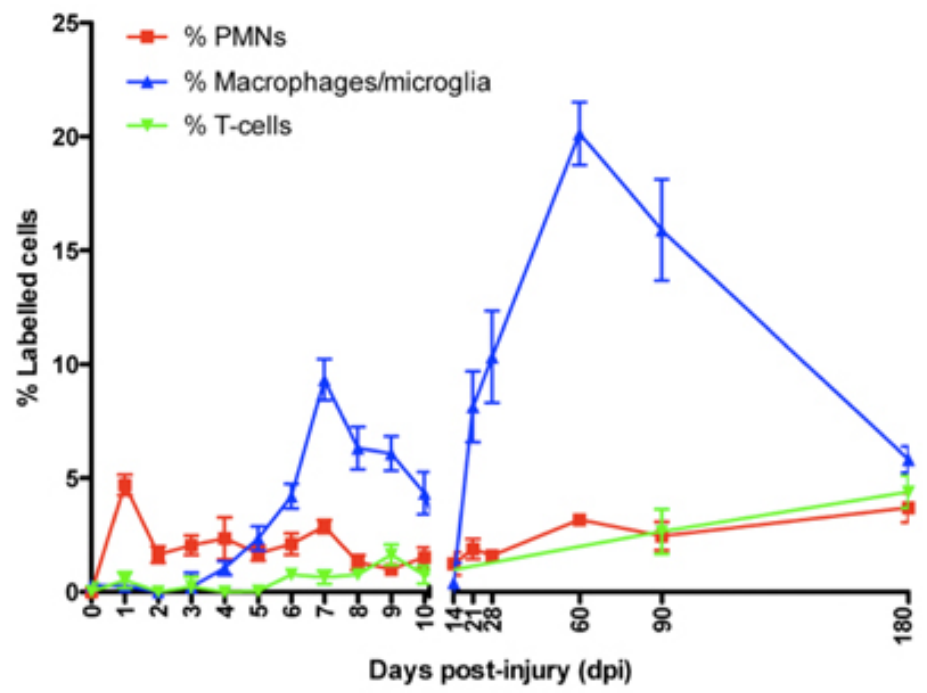

Figure 4. A timecourse of cellular inflammation in the spinal cord following a moderate $(200 \mathrm{kd})$ contusion injury at T9. As assessed by flow cytometry, numbers of PMNs, ED $1^{+}$macrophages/microglia, and $\mathrm{CD}^{+} \mathrm{T}$-cells peaked acutely $(1,7$, and $9 \mathrm{dpi}$, respectively) and persisted chronically in the injured spinal cord. $n=3$ to 5 per group, mean \pm SEM.

\section{Discussion}

The use of flow cytometry to quantify immune cells in the CNS is complicated by lipid/myelin content and debris. In addition to interfering with antibody binding and specificity, myelin debris can have similar size and granulation properties to immune cells, decreasing measurement sensitivity and accuracy ${ }^{8}$. The novel cell preparation method described here is effective in removing myelin debris and thereby improves the sensitivity of flow cytometry to detect changes in cellular inflammation in the injured spinal cord. For instance, the removal of myelin debris by this method enhanced detection of immune cells compared to enzymatic dissociation alone.Critically, novel usage of this method allowed the first characterization of acute and chronic cellular inflammation after SCI to include a complete time course for PMNs, macrophages/microglia, and T-cells up to $180 \mathrm{dpi}$, and identified a novel second phase of cellular inflammation. These important findings of a multiphasic component of neuroinflammation may be critical for the design and implementation of rational therapeutic treatment strategies, including both cell-based and pharmacological interventions for SCI.

The OptiPrep gradient system has been used to separate debris from cells in blood ${ }^{9}$, or purify neurons from the brain for cell culture purposes ${ }^{10}$; however, we have modified and advanced this method system to separate myelin debris from spinal cord dissociated cells, and allowed rapid and more accurate quantification of cellular inflammation by flow cytometry. This method together with flow cytometry is likely to provide significant advancement in inflammation research after CNS injuries or pathological conditions, as most studies have only characterized cellular inflammation in the CNS using morphological and histological techniques that are not cell-type specific or cannot always provide true quantitative assessment. Furthermore, a few recent studies have attempted to quantify immune cells in the injured spinal cord by flow cytometry using fresh dissociated cord tissue ${ }^{11,12}$ or cells separated by the Percoll gradient system ${ }^{13}$; however, these studies have demonstrated either low cell yields or insensitive detection of immune cells.

In contrast, the OptiPrep gradient cell preparation method has provided for the first time, sensitive and reliable quantitative assessment by flow cytometry to changes of cellular inflammation in response to graded injury severity and over an extended timecourse up to $180 \mathrm{dpi}$. The sensitivity and reliability of flow cytometric analysis also depend on the specificity of antibodies used to detect specific immune cell types, as flow cytometry does not permit morphological criteria to further distinguish cell types. The specificity of antibodies for PMNs, macrophages/ microglia or T-tells has been tested thoroughly in flow cytometry for peritoneal PMNs and alveolar macrophages in culture and cells in the injured spinal cord tissue ${ }^{1,2}$. Together with the OptiPrep gradient system, these antibodies used for flow cytometry have contributed to the generation of a temporal and quantitative analysis of cellular inflammation that has provided new insight into the dynamics of the SCI microenvironment, and identified for the first time an extended multiphasic response of cellular inflammation. This multiphasic response of cells after SCI may be important to investigate the role of specific cell types present at specific times after injury or generate therapeutic interventions against specific cell types that can aggravate injuries. Furthermore, these findings may assist in the development of an appropriate rationale for the optimal drugor cell-based interventions for SCl.

\section{Disclosures}

No conflicts of interest declared. 


\section{Acknowledgements}

We thank Rebecca Nishi, M.S., and the technicians of the Christopher \& Dana Reeve Foundation Animal Core Facility at UC Irvine for their help in animal surgery. We also thank Gabriella Funes, Usha Nekanti and Denisse Moreno for technical assistance, manuscript and video shoot preparations and animation. This research was supported by NINDS (RO1 NS43428-01 to AJA), the Paralysis Project of America (PPA-32574 to HXN), and California Institute for Regenerative Medicine (CIRM) Stem Cell Training Award T1-00008 to HXN. KDB was supported by the training program in molecular and cellular neuroscience at UC Irvine (T32 NS007444).

\section{References}

1. Beck, K.D., et al. Quantitative analysis of cellular inflammation after traumatic spinal cord injury: evidence for a multiphasic inflammatory response in the acute to chronic environment. Brain 133, 433-447 (2010).

2. Nguyen, H.X., Galvan, M.D. \& Anderson, A.J. Characterization of early and terminal complement proteins associated with polymorphonuclear leukocytes in vitro and in vivo after spinal cord injury. J Neuroinflammation 5, 26 (2008).

3. Saville, L.R., et al. A monoclonal antibody to CD11d reduces the inflammatory infiltrate into the injured spinal cord: a potential neuroprotective treatment. J Neuroimmunol 156, 42-57 (2004).

4. Popovich, P.G., Wei, P. \& Stokes, B.T. Cellular inflammatory response after spinal cord injury in Sprague-Dawley and Lewis rats. J Comp Neurol 377, 443-464 (1997).

5. Popovich, P.G., et al. Depletion of hematogenous macrophages promotes partial hindlimb recovery and neuroanatomical repair after experimental spinal cord injury. Exp Neurol 158, 351-365 (1999).

6. Jones, T.B., Hart, R.P. \& Popovich, P.G. Molecular control of physiological and pathological T-cell recruitment after mouse spinal cord injury. $J$ Neurosci 25, 6576-6583 (2005).

7. Kigerl, K.A., McGaughy, V.M. \& Popovich, P.G. Comparative analysis of lesion development and intraspinal inflammation in four strains of mice following spinal contusion injury. J Comp Neurol 494, 578-594 (2006).

8. Lipton, H.L., Kallio, P. \& Jelachich, M.L. Simplified quantitative analysis of spinal cord cells from Theiler's virus-infected mice without the requirement for myelin debris removal. $J$ Immunol Methods 299, 107-115 (2005).

9. Bagamery, K., Kvell, K., Landau, R. \& Graham, J. Flow cytometric analysis of CD41-labeled platelets isolated by the rapid, one-step OptiPrep method from human blood. Cytometry A 65, 84-87 (2005).

10. Majd, S., Zarifkar, A., Rastegar, K. \& Takhshid, M.A. Culturing adult rat hippocampal neurons with long-interval changing media. Iran Biomed J 12, 101-107 (2008).

11. Tjoa, T., et al. The use of flow cytometry to assess neutrophil infiltration in the injured murine spinal cord. J Neurosci Methods $129,49-59$ (2003).

12. Gonzalez, R., Glaser, J., Liu, M.T., Lane, T.E. \& Keirstead, H.S. Reducing inflammation decreases secondary degeneration and functional deficit after spinal cord injury. Exp Neurol 184, 456-463 (2003).

13. Stirling, D.P. \& Yong, V.W. Dynamics of the inflammatory response after murine spinal cord injury revealed by flow cytometry. $J$ Neurosci Res 86, 1944-1958 (2008). 